\section{LACTANTES EM TRATAMENTO MEDICAMENTOSO DA REDE PÚBLICA DE SAÚDE}

\author{
Breastfeeding women under medication treatment in the public \\ health network
}

Lactantes en terapia medicamentosa de la red pública de salud
Artigo Original

Viviane Muniz da Silva Fragoso(1) Elizabeth Domingues da Silva ${ }^{(2)}$ Josiane Monsores Mota ${ }^{(2)}$
Objetivo: Analisar os medicamentos das lactantes em tratamento na rede pública de saúde e as ações envolvidas. Métodos: Estudo transversal, quantitativo e descritivo realizado com 100 lactantes selecionadas através de amostragem não probabilística e por conveniência no Hospital Municipal de Duque de Caxias-RJ, no ano de 2012. Aplicou-se um questionário contendo as seguintes variáveis: informações sociodemográficas, medicamentos prescritos, efeitos indesejados nos lactentes, e profissionais envolvidos nas orientações quanto ao uso desses medicamentos. Analisaram-se os dados através da estatística descritiva, a partir de frequências absolutas e relativas. Resultados: Identificou-se que 46\% $(n=46)$ das lactantes tinham entre 21 e 30 anos, 54\% $(n=54)$ eram primíparas, 52\% $(n=52)$ tinham ensino fundamental completo e $72 \%(\mathrm{n}=72)$ receberam o acompanhamento pré-natal. Verificouse que $78 \%(n=78)$ faziam uso de algum tipo de medicamento, dentre eles, um percentual significativo de analgésicos/anti-inflamatórios não esteroidais, com 61,54\% ( $n=48)$ das lactantes. Todos os medicamentos prescritos estavam na categoria de uso compatível com a amamentação. Constatou-se presença de sintomas indesejados em 19,2\% das lactantes $(n=15)$. Das lactantes em terapia medicamentosa, 76,92 \% $(n=60)$ tiveram orientação durante o tratamento, sendo $55 \%(n=33)$ por médicos e $45 \%(n=27)$ por enfermeiros. Nesta pesquisa, $100 \%$ das lactantes ficaram satisfeitas com o aprendizado. Conclusão: Observou-se número elevado de lactantes da amostra fazendo uso de medicamentos, todos compatíveis com a amamentação. Ressalta-se a participação restrita da equipe multidisciplinar nas orientações.

Descritores: Aleitamento Materno; Tratamento Medicamentoso; Sistema Único de Saúde.

\section{ABSTRACT}

Objective: To analyse the medications used by breastfeeding women treated in the public health network, and correlated actions. Methods: Cross-sectional, quantitative and descriptive study carried out with 100 breastfeeding women, recruited through nonprobabilistic convenience sampling, at the Municipal Hospital of Duque de Caxias, $R J$, in 2012. A questionnaire was applied containing the following variables: prescribed medications, unwanted effects in nursing infants, and professionals involved in guidance on the edication. The data was analysed through descriptive statistics, based on absolute and relative frequencies. Results: It was found that $46 \%(n=46)$ of the breastfeeding women were aged 21 to 30 years, $54 \%(n=54)$ were primiparae, $52 \%(n=52)$ had complete fundamental level, and $72 \%(n=72)$ received prenatal care. It was verified that $78 \%(n=$ 78) of the sample were receiving some type of medicine and, among these, a significant percentage of nonsteroidal analgesic/anti-inflammatory medication, with $61.54 \%(n=48)$ of the breastfeeding women. All the prescribed medicines were in the category of compatible use with breastfeeding. The incidence of some unwanted symptoms was evidenced in $19.2 \%$ $(n=15)$ of the breastfeeding women. Among the women undergoing medication therapy, $76.92 \%(n=60)$ received guidance during treatment, 55\% ( $n=33)$ by doctors and $45 \%$ $(n=27)$ by nurses. In this research, 100\% of the breastfeeding women were satisfied with the acquired knowledge. Conclusion: It was noted a high percentage of breastfeeding women in the sample taking medicines, all compatible with breastfeeding. It stands out the limited engagement of the multidisciplinary team in the orientations.

Descriptors: Breast Feeding; Medication Treatment; Unified Health System.
1) Fundação Oswaldo Cruz - FIOCRUZ Rio de Janeiro (RJ) - Brasil

2) Universidade do Grande Rio UNIGRANRIO - Rio de Janeiro (RJ) Brasil
Recebido em: 26/03/2013 Revisado em: 23/05/2013 Aceito em: 24/01/2014 


\section{RESUMEN}

Objetivo: Analizar los medicamentos de las lactantes asistidas en la red pública de salud y las acciones involucradas. Métodos: Estudio transversal, cuantitativo y descriptivo con 100 lactantes seleccionadas a través de muestreo no probabilístico y por conveniencia en el Hospital Municipal de Duque de Caxias-RJ en el año de 2012. Se aplico un cuestionario con las siguientes variables: informaciones sociodemograficas, medicamento prescrito, efectos no deseados en las lactantes y profesionales involucrados con las orientaciones sobre el uso de estos medicamentos. Los datos fueron analizados a través de estadística descriptiva a partir de frecuencias absolutas y relativas. Resultados: Se identifico que el 46\% $(n=46)$ de las lactantes tenían entre 21 y 30 años, el $54 \%(n=54)$ eran primíparas, el $52 \%$ $(n=46)$ tenían la educación secundaria completa y el $72 \%(n=72)$ realizaron el prenatal. Se verifico que el $78 \%(n=78)$ usaban algún tipo de medicamento, entre ellos, un porcentaje significativo de analgésicos/antiinflamatorios no esteroideo en el 61,54\% $(n=48)$ de las lactantes. Todos los medicamentos prescritos eran de uso compatible con la lactancia. Se constató la presencia de síntomas no deseados en el 19,2\% $(n=15)$ de las lactantes. De las que estaban en terapia medicamentosa el 76,92\% $(n=60)$ tuvieron orientación durante el tratamiento siendo el 55\% $(n=33)$ por médicos y el 45\% $(n=27)$ por enfermeros. En esta investigación el $100 \%$ de las lactantes se quedaron satisfechas con el aprendizaje. Conclusión: Se observó un elevado número de lactantes de la muestra en terapia medicamentosa compatible con la lactancia. Se destaca la participación restricta del equipo multidisciplinario en las orientaciones.

Descriptores: Lactancia Materna; Quimioterapia; Sistema Único de Salud.

\section{INTRODUÇÃO}

A Organização Mundial de Saúde ressalta que o leite materno é o alimento ideal para o desenvolvimento e o crescimento saudável dos lactentes, e deve ser introduzido logo após o nascimento. A amamentação exclusiva é recomendada até os seis meses de idade e deve continuar até os dois anos de idade ou mais, junto com outros alimentos ${ }^{(1)}$.

No Brasil, o Ministério da Saúde afirma que o aleitamento materno exclusivo até os seis meses de idade pode evitar, anualmente, 1,3 milhões de mortes de crianças menores de cinco anos. O leite humano é composto de nutrientes adequados que oferecem vantagens imunológicas, cognitivas e psicoafetivas, além de prolongar o período entre as gestações e diminuir a incidência de algumas doenças na mulher ${ }^{(2,3)}$.

Após o parto, o uso de medicamentos é frequente entre as lactantes, devido à necessidade de tratamento para combater infecções, depressão, além das doenças crônicas que requerem medicamentos de uso contínuo ${ }^{(4-6)}$. Sabe-se que mais de $50 \%$ das mulheres que estão amamentando usam algum tipo de medicamento ${ }^{(7,8)}$.

Geralmente, a concentração do fármaco que alcança o leite materno é reduzida, apresentando baixa relevância para o lactente ${ }^{(9)}$. No entanto, muitas mulheres são aconselhadas a interromper a amamentação em razão do uso de algum medicamento ${ }^{(10,11)}$, impedindo que a mãe e a criança desfrutem dos benefícios do aleitamento, levando ao desmame. Entretanto, a transferência do fármaco do plasma para o leite materno pode resultar na ingestão e absorção do medicamento pelo trato gastrointestinal, apresentando risco potencial ao lactente ${ }^{(1,3)}$.

Em geral, os profissionais de saúde que atuam prescrevendo medicamentos para lactantes devem se basear na relação risco/benefício e conhecer as categorias de risco das drogas, que vão desde as mais seguras até as contraindicadas $^{(12)}$. Porém, a carência de informações e a complexidade dos diversos fatores que determinam a escolha de um medicamento para uso durante a lactação reforçam a preocupação sobre a automedicação e a interrupção da amamentação por orientação dos profissionais de saúde ${ }^{(13-15)}$. A amamentação somente deverá ser interrompida se existirem evidências de que a droga usada pela lactante é nociva para o lactente, ou quando não houver informações a respeito e a droga não puder ser substituída por outra que seja compatível com a amamentação ${ }^{(3)}$.

Os profissionais de saúde têm um papel importante na promoção da amamentação durante o tratamento farmacológico. A atuação desses profissionais nos estabelecimentos de saúde oferece o suporte necessário às mães para a manutenção do aleitamento materno e a terapia medicamentosa correta, reduzindo os riscos ao lactente ${ }^{(15)}$. O profissional de saúde deve buscar informações atualizadas para avaliar os riscos e os benefícios do uso de uma determinada droga em uma mulher que está amamentando. O cuidado na escolha do medicamento para tratar a lactante permitirá a continuidade na amamentação, sem interrupção e com segurança ${ }^{(1)}$.

Visando contribuir para a promoção da saúde das lactantes e dos lactentes do Hospital Municipal de Duque de Caxias-RJ, e seguindo os objetivos propostos pelo Ministério da Saúde no manual "Amamentação e uso de medicamentos e outras substâncias"(2) e no Programa Nacional do Aleitamento Materno ${ }^{(16)}$, este estudo objetivou analisar os medicamentos das lactantes em tratamento na rede pública de saúde e as ações envolvidas.

\section{MÉTODOS}

Estudo transversal, descritivo e de caráter quantitativo, realizado no Hospital Municipal Maternidade Xerém, em 
Duque de Caxias-RJ, que presta serviços de saúde pública às lactantes do município.

A amostra não probabilística e por conveniência envolveu 100 lactantes na faixa etária de 15 a 40 anos, selecionadas nas consultas de retorno pós-parto no hospital, no período de maio a junho de 2012.

Após a seleção, a coleta dos dados ocorreu mediante aplicação de um questionário $\operatorname{adaptado}^{(17)}$ contendo perguntas fechadas, padronizadas e de fácil compreensão, direcionadas a cada lactante entrevistada, sem limite de tempo para as respostas. As variáveis analisadas neste estudo foram: informações sociodemográficas, medicamentos prescritos, efeitos indesejados nos bebês, e profissionais envolvidos na orientação das lactantes. Para minimizar os erros de coleta, os pesquisadores que aplicaram os questionários foram previamente treinados.

Os medicamentos prescritos no presente estudo foram comparados com a sua categoria de risco, a partir do manual do Ministério da Saúde ${ }^{(2)}$ que identifica a compatibilidade ou não de drogas com a amamentação.

Aplicou-se a estatística descritiva para análise dos dados, expressos por frequências absolutas e percentuais através do programa Estat $\mathrm{D}^{+}$.

A pesquisa seguiu a orientação do Conselho Nacional de Saúde através da Resolução n ${ }^{0} 196 / 96^{(19)}$. A pesquisa recebeu aprovação do Comitê de Ética em Pesquisa da Universidade do Grande Rio (parecer n ${ }^{\circ}$ CAEE. 00750512.1.0000.5283).
Antes de iniciar o estudo, as voluntárias, bem como os responsáveis legais das lactantes menores de idade, assinaram o Termo de Consentimento Livre e Esclarecido.

\section{RESULTADOS}

A caracterização geral da amostra evidenciou que, das lactantes entrevistadas, $46 \% \quad(n=46)$ estavam na faixa etária entre 21 e 30 anos, $54 \%(n=54)$ tinham até um filho (primíparas), $52 \%(\mathrm{n}=52)$ estudaram até o ensino fundamental, $81 \%(\mathrm{n}=81)$ responderam que não desenvolviam nenhuma ocupação profissional e $72 \%$ $(\mathrm{n}=72)$ realizaram pré-natal (Tabela I).

Quanto às lactantes que utilizaram medicamentos após o parto, $78 \%(\mathrm{n}=78)$ confirmaram o uso de algum tipo de medicamento. Desse percentual, 61,54\% $(n=48)$ dos medicamentos referiam-se a analgésicos e anti-inflamatórios não esteroidais. Todos os medicamentos prescritos para as lactantes encontravam-se na categoria de uso compatível com a amamentação, inclusive os antibióticos e antihipertensivos (Tabela II).

Sobre os possíveis sintomas indesejados nos lactentes, 19,2\% ( $\mathrm{n}=15)$ apresentaram pelo menos um após a ingestão materna de medicamentos, destacando-se a cólica (Tabela III). Nessa análise, levou-se em consideração o fato de os sintomas observados nos lactentes serem ou não provenientes dos medicamentos ingeridos pelas mães, uma vez que não

Tabela I - Distribuição das lactantes quanto à faixa etária, número de filhos, nível de escolaridade, ocupação e acompanhamento durante o pré-natal, no Hospital Municipal Maternidade Xerém. Duque de Caxias-RJ, 2012.

\begin{tabular}{|c|c|c|}
\hline & $\begin{array}{c}\text { Lactantes } \\
(\mathrm{n}=100)\end{array}$ & $\%$ \\
\hline \multicolumn{3}{|l|}{ Faixa etária (anos) } \\
\hline $15-20$ & 40 & 40 \\
\hline $21-30$ & 46 & 46 \\
\hline $31-40$ & 14 & 14 \\
\hline \multicolumn{3}{|l|}{ Número de filhos } \\
\hline Até 1 & 54 & 54 \\
\hline 2 ou 3 & 33 & 33 \\
\hline 4 ou mais & 13 & 13 \\
\hline \multicolumn{3}{|l|}{ Escolaridade } \\
\hline Ensino Fundamental $\left(1^{\circ}\right.$ ao $5^{\circ}$ ano $)$ & 24 & 24 \\
\hline Ensino Fundamental ( $6^{\circ}$ ao $9^{\circ}$ ano $)$ & 28 & 28 \\
\hline Ensino Médio & 46 & 46 \\
\hline Ensino Superior & 2 & 2 \\
\hline \multicolumn{3}{|l|}{ Ocupação } \\
\hline Não & 81 & 81 \\
\hline Sim & 19 & 19 \\
\hline \multicolumn{3}{|c|}{ Acompanhamento durante o período gestacional } \\
\hline $\operatorname{Sim}$ & 72 & 72 \\
\hline Não & 28 & 28 \\
\hline
\end{tabular}


Tabela II - Distribuição das lactantes em relação ao percentual das respostas para o uso ou não de medicamentos, agrupados em classes terapêuticas, no Hospital Municipal Maternidade Xerém. Duque de Caxias-RJ, 2012.

\begin{tabular}{lcc}
\hline Classes Terapêuticas & $\begin{array}{c}\text { Lactantes } \\
(\mathbf{n = 7 8 )}\end{array}$ & $\mathbf{\%}$ \\
\hline Analgésicos e Anti-inflamatórios Não Esteroidais & & 61,54 \\
Sim & 48 & 38,46 \\
Não & 30 & \\
Antibióticos & & 24,36 \\
Sim & 19 & 75,64 \\
Não & 59 & \\
Vitaminas & & 20,51 \\
Sim & 16 & 79,49 \\
Não & 62 & \\
Antiespasmódicos & & 12,82 \\
Sim & & 87,18 \\
Não & 10 & \\
Anti-hipertensivos & 68 & 6,41 \\
Sim & & 93,59 \\
Não & 5 & \\
\hline
\end{tabular}

Tabela III - Distribuição dos sintomas observados nos lactentes após o uso de medicamentos nas lactantes no Hospital Municipal Maternidade Xerém. Duque de Caxias-RJ, 2012.

\begin{tabular}{lcc}
\hline Possíveis Sintomas Indesejados & $\begin{array}{c}\text { Lactentes } \\
(\mathbf{n = 1 5})\end{array}$ & $\mathbf{\%}$ \\
\hline Cólicas & 5 & 33,33 \\
Sonolência & 1 & 6,67 \\
Êmese & 4 & 26,67 \\
Insônia & 2 & 3,33 \\
Outros* & 5 & 33,33 \\
\hline
\end{tabular}

*Febre, diarreia e refluxo

se realizaram testes para verificar. Considerando-se somente as lactantes cujos filhos apresentaram possíveis sintomas indesejados, $40 \%(\mathrm{n}=6)$ interromperam o tratamento. Desse percentual, somente $33,3 \%(\mathrm{n}=2)$ trocaram o medicamento - um em razão de alergia ao medicamento e o outro por motivo não referido pela mãe.

Ao pesquisar se as 78 lactantes em tratamento medicamentoso receberam orientações sobre o uso dos medicamentos durante a amamentação, constatou-se que $76,92 \%(n=60)$ deram respostas afirmativas. Das lactantes orientadas quanto ao uso de medicamentos, $55 \%(\mathrm{n}=33)$ relataram que médicos as orientaram e $45 \%(n=27)$ disseram que os enfermeiros transmitiram informações sobre o uso dos fármacos prescritos. Elas afirmaram não ter recebido orientação de outros profissionais de saúde, como farmacêuticos e técnicos de enfermagem.

\section{DISCUSSÃO}

O período do aleitamento materno está relacionado com alguns fatores positivos, como idade e nível de escolaridade, e negativos, como ausência do apoio da família, inserção da mulher no mercado de trabalho e realização do parto cesárea ${ }^{(19,20)}$.

Do grupo de lactantes entrevistadas na presente pesquisa, verificou-se predominância das mulheres com idade inferior a 30 anos. Em estudo realizado no Hospital Público do Distrito Federal, em Brasília, 76,47\% das 
lactantes possuíam idade entre 20 e $29 \operatorname{anos}^{(20)}$. As mulheres mais jovens, principalmente abaixo dos 20 anos, período em que a maturidade fisiológica e emocional não foi completamente alcançada, apresentam uma tendência de amamentar por período mais curto ${ }^{(21)}$. Por outro lado, mulheres com idade mais avançada amamentam os seus filhos por mais tempo ${ }^{(22,23)}$.

Analisou-se o percentual de lactantes que tinha ou não uma ocupação profissional. Do total, $81 \%$ estavam desempregadas. Em estudo realizado no Hospital Público do Distrito Federal, em Brasília, 52,94\% das lactantes trabalhavam fora de casa ${ }^{(20)}$. Uma pesquisa mostrou que mães que trabalham dentro de casa amamentam com mais facilidade os seus filhos ${ }^{(24)}$. Contudo, outro trabalho relatou que a amamentação pode aumentar entre mães que trabalham fora de casa ${ }^{(25)}$. Outro ponto a ser destacado é que as mulheres que trabalham fora do lar apresentam mais cedo a mamadeira aos seus filhos, por causa da preocupação com a possibilidade de a criança não se adaptar à alimentação $\operatorname{artificial}^{(26)}$.

Com relação ao nível de escolaridade, a presente pesquisa revelou que apenas $2 \%$ tinham o nível superior completo. A maior parte possuía ensino fundamental ou ensino médio completo, e nenhuma era analfabeta. Esse resultado corrobora com um estudo semelhante, no qual se verificou que o maior percentual de lactantes possuía o ensino médio completo ${ }^{(20)}$. Isso pode prejudicar o aprendizado no pré-natal, dificultando ou facilitando a amamentação, já que o nível escolar mais alto pode aumentar a inserção das mulheres no mercado de trabalho ${ }^{(27)}$.

A realização do pré-natal pode reduzir a mortalidade através do diagnóstico e tratamento de doenças acometidas na mãe, a fim de evitar complicações. Além disso, o pré-natal aumenta o conhecimento e a confiança da mãe sobre a amamentação ${ }^{(28,29)}$. Observou-se que $72 \%$ das puérperas realizaram pré-natal. Isso pode ser confirmado no estudo que revelou a orientação sobre o aleitamento materno de $64,71 \%$ das lactantes durante o pré-natal ${ }^{(20)}$. A ausência de acompanhamento médico durante a gestação pode representar riscos para as vidas de mães e bebês, já que o seu objetivo é prevenção, orientação, esclarecimento e diagnóstico de qualquer alteração da saúde da gestante e/ ou do bebê.

A ausência de informações sobre dados de transferência de novas drogas para o leite materno, além da segurança, são fatores que dificultam a utilização dessas substâncias nas lactantes ${ }^{(1)}$. Verificou-se que $78 \%$ das lactantes utilizaram medicamentos segundo prescrição médica. A classe terapêutica mais prescrita foi a dos analgésicos e anti-inflamatórios não esteroidais, seguida dos antibióticos, vitaminas, antiespasmódicos e anti-hipertensivos. Após a análise da categoria de risco segundo o Ministério da
Saúde ${ }^{(1)}$, observou-se que todos os medicamentos prescritos às lactantes encontravam-se dentro da classificação de uso compatível com a amamentação. Em contrapartida, outro estudo mostrou que medicamentos prescritos às lactantes apresentavam efeitos desconhecidos e significativos em alguns lactentes ${ }^{(13)}$. Um estudo atribuiu esse fato à desinformação (em especial, de médicos), ao conflito de informações das bulas dos medicamentos e às poucas evidências científicas quanto ao uso destes durante o aleitamento $^{(12)}$.

Outro fato evidenciado neste trabalho foi que, das 78 mulheres que receberam medicamentos, $76,92 \%$ foram orientadas quanto ao uso dos fármacos por médicos ou enfermeiros, sendo mencionada pelas lactantes a ausência de orientação por parte de outros profissionais de saúde, como os farmacêuticos.

O farmacêutico é o profissional que trabalha com medicamentos dentro do hospital, e a falta desse funcionário na orientação sobre o uso correto dos medicamentos pode gerar prejuízos à saúde da lactante e do lactente. A interação direta do farmacêutico com a lactante traz benefícios quanto ao uso racional do medicamento e leva a resultados direcionados à melhoria da qualidade de $v i^{(30)}$. O prejuízo do uso incorreto de medicamentos vai além da lactante e pode alcançar a criança, mediante a transferência da droga para o leite materno e a absorção pelo lactente, resultando em efeitos indesejados ${ }^{(31)}$. Um estudo sugeriu que a orientação através de profissionais do Programa de Saúde da Família e de Agentes Comunitários de Saúde realizando visitas domiciliares não elimina a importância da consulta com o farmacêutico e o médico ${ }^{(15)}$.

Na presente pesquisa, pôde-se analisar que, após o uso de fármacos, ocorreram relatos de sintomas indesejados em 19,2\% dos bebês. Ressalta-se, entretanto, que as drogas prescritas eram de uso compatível com a amamentação ${ }^{(2)}$. Além disso, os prescritores escolheram medicamentos cujos efeitos são descritos na literatura, pouco excretados no leite materno e que não oferecem risco para saúde da criança, como já abordado em outro trabalho ${ }^{(14)}$. Para a confirmação da associação desses sintomas com os medicamentos prescritos, testes específicos faziam-se necessários.

Os profissionais de saúde têm um papel fundamental na promoção da amamentação e na orientação sobre os medicamentos que trazem benefícios terapêuticos à saúde materna, sem riscos para a saúde do lactente ${ }^{(32)}$, sendo primordial a compreensão das lactantes sobre a relevância do aleitamento materno e o uso cauteloso de medicamentos durante a amamentação ${ }^{(33)}$. Assim, a continuidade de pesquisas que envolvem a prescrição de medicamentos para as lactantes e o acompanhamento das reações nos lactentes devem ser estimulados. 


\section{CONCLUSÃO}

Observou-se um número elevado de lactantes fazendo uso de medicamentos, todos compatíveis com a amamentação. Ressalta-se a participação restrita da equipe multidisciplinar nas orientações.

\section{AGRADECIMENTOS}

À Dra Tânia Maria Lemos Mouço, Professora de Atenção Farmacêutica do Curso de Farmácia da Universidade do Grande Rio e Conselheira do Conselho Regional de Farmácia do Rio de Janeiro, pelo apoio logístico na pesquisa e ao Dr Frederico Alan de Oliveira Cruz, Professor Adjunto da Universidade Federal Rural do Rio de Janeiro, pelo apoio técnico neste estudo.

\section{REFERÊNCIAS}

1. World Health Organization - WHO. Breastfeeding [acesso em 2014 Ago 12]. Disponível em: http://www. who.int/topics/breastfeeding/en/

2. Ministério da Saúde (BR), Secretaria de Atenção à Saúde, Departamento de Ações Programáticas e estratégicas: amamentação e uso de medicamentos e outras substâncias. Brasília: Ministério da Saúde; 2010.

3. Fundo das Nações Unidas para a Infância - UNICEF [Internet]. Aleitamento materno [acesso em 2011 Set 12]. Disponível em: http://www.unicef.org/brazil/pt/ activities_10003.htm

4. Amir L, Pirotta M, Raval M. Breastfeeding-evidence based guidelines for the use of medicines. Aust Fam Physician. 2011;40(9):684-90

5. Schirm E, Schwagermann M, Tobi H, de Jong-van den Berg LTW. Drug use during breastfeeding: a survey from the Netherlands. Eur J Clin Nutr. 2004;58(2):38690.

6. Jayawickrama H, Amir LH, Pirotta MV. GPs' decisionmaking when prescribing medicines for breastfeeding women: content analysis of a survey. BMC Res Notes. 2010;3(82):1-9

7. Hussainy SY, Dermele N. Knowledge, attitudes and practices of health professionals and women towards medication use in breastfeeding: a review. Inter Breastfeed J. 2011;6(11):1-16.

8. Fortinguerra F, Clavenna A, Bonati M. Psychotropic drug use during breastfeeding: a review of the evidence. Pediatrics. 2009;124(4):e547-6.
9. Almeida JLJ, Kubo F, Silva CAA, Issler H. Uso de antiinflamatórios não-hormonais durante a amamentação: quais podem ser utilizados? Rev Paul Pediatr. 2006;24(2):171-9.

10. Nice FJ, Luo AC. Medications and breast-feeding: current concepts. J Am Pharm Soc. 2012;52(1):86-94

11. Berlin Junior CM, Van Den Anker JN. Saftey during breastfeeding: drugs, foods environmental chemicals and maternal infections. Semin Fetal Neonatal Med. 2013;18(1):13-8.

12. Chaves RG, Lamounier JA, César CC. Medicamentos e amamentação: atualização e revisão aplicadas à clínica materno-infantil. Rev Paul Pediatr. 2007;25(3):27688.

13. Chaves RG, Lamounier JA. Uso de medicamentos durante a lactação. J Pediatr (Rio J.). 2004;80(5):18998.

14. Chaves RG, Lamounier JA, César CC. Automedicação em lactantes e sua influência sobre a duração do aleitamento materno. J Pediatr (Rio J.). 2009;85(2):12934.

15. Azeredo CM, Maia TM, Rosa TCA, Silva FF, Cecon PR, Cotta RMM. Percepção de lactantes e profissionais de saúde sobre o aleitamento materno: encontros e desencontros. Rev Paul Pediatr. 2008;26(4):336-44.

16. Alencar SMS. A Política nacional de aleitamento materno. In: $\mathrm{O}$ aleitamento materno no contexto atual: políticas, práticas e bases científicas. São Paulo: Sarvier; 2008. p. 70-101.

17. Del Ciampo LA, Ferraz IS, Daneluzzi JC, Ricco RG, Martinelli Junior CE. Aleitamento materno e uso de medicamentos durante a lactação. Rev Paul Pediatr. 2007;25(4):355-57.

18. Ministério da Saúde (BR), Conselho Nacional de Saúde. Resolução no 196, de 10 de outubro de 1996. Aprova as diretrizes e normas regulamentadoras de pesquisas envolvendo seres humanos. Diário Oficial da União. Brasília; 1996.

19. Roig AO, Martínez MR, García JC, Hoyo SP, Navidad GL, Álvarez JCF, et al. Fatores associados ao abandono do aleitamento materno durante os primeiros seis meses de vida. Rev Latinoam Enferm. 2010;18(3):80-5.

20. Fragoso APR, Fortes RC. Fatores associados à prática do aleitamento materno entre lactantes de um hospital público do Distrito Federal. J Health Sci Inst. 2011;29(2):114-8. 
21. Maciel APP, Gondim APS, Silva AMV, Barros FC, Barbosa GL, Albuquerque KC, et al. Conhecimento de gestantes e lactantes sobre aleitamento materno exclusivo. Rev Bras Promoç Saúde. 2013;26(3):311-7.

22. Faleiros FTV, Trezza EMC, Carandina L. Aleitamento materno: fatores de influência na sua decisão e duração. Rev Nutr. 2006;19(5):623-30.

23. Queirós PS, Oliveira LRB, Martins CA. Elementos que interferem na amamentação exclusiva: percepções de lactantes. Rev Salud Pública. 2009;13(2):6-14.

24. Azevedo DS, Reis ACS, Freitas LV, Costa PB, Pinheiro PNC, Damasceno AKC. Conhecimento de primíparas sobre os benefícios do aleitamento materno. Rev RENE. 2010;11(2):53-62.

25. Escobar AMU, Ogawa AR, Hiratsuka M, Kawashita MY, Teruya PY, Grisi S, et al. Aleitamento materno e condições socioeconômico-culturais: fatores que levam ao desmame precoce. Rev Bras Saúde Matern Infant. 2002;2(3):253-61.

26. Issler H, Douek PC, André LM, Goldstein SR, Issa LJ, Fujinami PI, et al. Fatores socioculturais do desmame precoce: estudo qualitativo. Pediatria. 2010; 32(2):11320.

27. Damião JJ. Influência da escolaridade e do trabalho maternos no aleitamento materno exclusivo. Rev Bras Epidemiol. 2008;11(3):442-52.

28. Nunes PHC, Pereira BMG, Nominato JCS, Albuquerque EM, Silva LFN, Castro IRS, et al. Intervenção farmacêutica e prevenção dos eventos adversos. Rev Bras Ciênc Farm. 2008; 44(4):691-9.
29. Christoffel MM, Votto MG, Allevato CG, Ambrósio MDV, Araújo AS. Práticas de amamentação de puérperas na consulta de enfermagem neonatal em uma unidade básica de saúde. REME Rev Min Enferm. 2009;13(2):202-8.

30. Consenso Brasileiro de Atenção Farmacêutica (BR), Organização Pan-Americana da Saúde. Atenção Farmacêutica no Brasil: "Trilhando Caminhos". Brasília; 2002.

31 Ribeiro MSS, Nunes RN, Silva CDC, Sudo EC, Mota DM, Coelho HLL. Medicamentos de risco para a gravidez e lactação comercializados no Brasil: uma análise de bulas. Acta Farm Bonaer. 2005;24(3):441-8.

32. Castro RA, Oliveira EM, França-Botelho AC. Aleitamento materno em área de abrangência do Programa de Saúde da Família. Rev Bras Promoç Saúde. 2009;22(1):30-5.

33. Saliba NA, Zina LG, Moimaz SAS, Saliba D. Frequência e variáveis associadas ao aleitamento materno em crianças com até 12 meses de idade no município de Araçatuba, São Paulo Brasil. Rev Bras Saúde Matern Infant. 2008;8(4):481-90.

\section{Endereço para correspondência:}

Viviane Muniz da Silva Fragoso

Laboratório de Inovações em Terapias, Ensino e

Bioprodutos

Instituto Oswaldo Cruz /IOC/FIOCRUZ

Avenida Brasil, 4365

Bairro: Manguinhos

CEP: 21040-360 - Rio de Janeiro - Rio de Janeiro - Brasil 
